UT-Komaba 01-02

hep-th/0106218

June, 2001

\title{
Fully Off-shell Effective Action and its Supersymmetry in Matrix Theory II
}

\author{
Y. Kazamal] and T. Muramatsu \\ Institute of Physics, University of Tokyo, \\ Komaba, Meguro-ku, Tokyo 153-8902 Japan
}

\begin{abstract}
In a previous work, we computed the fully off-shell effective action $\Gamma$ and the corresponding quantum-corrected supersymmetry (SUSY) transformation operator $\delta_{\epsilon}$ for the so-called source-probe configuration in Matrix theory at one loop at order 4 in the derivative expansion, and showed that they satisfy the SUSY Ward identity $\delta_{\epsilon} \Gamma=0$. In this article, starting from the most general form of $\Gamma$, we demonstrate that, conversely, given such $\delta_{\epsilon}$ the SUSY Ward identity determines $\Gamma$ uniquely to the order specified above. Our demonstration does not require the explicit knowledge of the quantum-corrected supersymmetry transformation and hence strongly suggests that the uniqueness property would persist to all orders in perturbation theory.
\end{abstract}

\footnotetext{
†kazama@hep3.c.u-tokyo.ac.jp

†tetsu@hep1.c.u-tokyo.ac.jp
} 


\section{Introduction}

This is a continuation of the previous investigations [1, 2] on the question of the power of supersymmetry (SUSY) in Matrix theory for M-theory [3, 4, 5].

Since its birth, Matrix theory has enjoyed numerous successes in a variety of contexts, for which we refer the reader to an assortment of review articles [6]. A simple yet fundamental question is "why ?" Although a number of appealing reasons were presented in the original proposal [3] and additional supportive arguments were subsequently supplied in [7, 8], it is fair to say that the precise reason is yet to be identified.

Undoubtedly, one of the key ingredients must be the high degree of SUSY present in the theory. Besides playing the crucial role of allowing the scattering states to exist, it supports a variety of stable brane configurations expected of a proper representation of M-theory. Furthermore, evidence has been accumulating [9, 10, 11, 12, 13, 14] that at least for some restricted configurations it appears to be powerful enough to control the dynamical details of the scattering. This is rather surprising since normally such a global symmetry can only give certain relations among correlation functions and cannot fix the scattering amplitudes.

Thus it is of great interest to know precisely how much is determined by SUSY. This requires investigation of the off-shell correlation functions as well, i.e. of the effective action without imposing the equations of motion. In contrast to the case with a small number of SUSY, the theory with maximal SUSY, such as Matrix theory under consideration, presents difficulties in answering to this question because the off-shell unconstrained superfield formalism is not known and one is forced to deal with the component formalism. In such non-covariant formulation, the SUSY algebra gets intimately intertwined with gauge symmetry and does not close without the aid of the equations of motion. Besides, the SUSY transformations for the effective action get non-trivial quantum corrections, which makes the analysis quite cumbersome.

Nevertheless, in previous works we have made some progress in understanding the role of SUSY for the fully off-shell effective action for the so-called the source-probe configuration, where a probe D-particle, with its position and the spin described respectively by the bosonic coordinates $r_{m}(\tau)$ and the fermionic coordinates $\theta_{\alpha}(\tau)$, interacts with a large number of source D-particles all sitting at the origin. First the precise form of the SUSY Ward identity, mixed with the gauge structure in essential ways, was derived together with closed form expressions for the quantum-corrected SUSY transformation laws [1]. This formalism was then explicitly applied to the simplest case, namely to the 
1-loop effective action at order 2 in the sense of the derivative expansion $\$$, and it was found that, with the knowledge of the SUSY transformation, the SUSY Ward identity completely fixed the form of the off-shell effective action to the order investigated. This example, however, was not so significant since at this order the higher order derivatives, such as acceleration etc., that do not vanish by the equation of motion nevertheless can be eliminated by integration by parts.

The full significance of our off-shell machinery becomes apparent at order 4, where complete elimination of higher derivatives is no longer possible. Since, unlike for order 2 , only a small portion of the fully off-shell 1-loop effective action $\Gamma^{(1)}$ was known at this order, we had to first complete its computation, including all the spin effects [2]. This was a horrendous task, involving manipulations of hundreds of terms in the intermediate stages. However, with judicious use of $S O(9)$ Fierz identities, including some new ones, the result turned out to be much simpler than was expected. To check this result as well as for its own interest, we also computed the quantum-corrected SUSY transformation operator $\delta_{\epsilon}$ up to the relevant order. Although their forms were embarrassingly complicated, we succeeded in verifying the Ward identity $\delta_{\epsilon}\left(\Gamma^{(0)}+\Gamma^{(1)}\right)=0$, where $\Gamma^{(0)}$ is the tree-level action.

The main purpose of the present work is to demonstrate that, conversely, given such $\delta_{\epsilon}$ our $\Gamma^{(1)}$ is in fact the unique solution to the SUSY Ward identity at one-loop at order 4. We shall show this in the following way: Let us write the most general effective action up to 1-loop as $\Gamma=\Gamma^{(0)}+\Gamma^{(1)}+\Delta \Gamma^{(1)}$, where $\Gamma^{(1)}$ is our previous result and $\Delta \Gamma^{(1)}$ is a possible arbitrary addition at order 4 . Then, expanding $\delta_{\epsilon}$ also to 1-loop as $\delta_{\epsilon}=\delta_{\epsilon}^{(0)}+\delta_{\epsilon}^{(1)}$, the Ward identity $\delta_{\epsilon} \Gamma=0$ is satisfied to the desired order if and only if $\delta_{\epsilon}^{(0)} \Delta \Gamma^{(1)}=0$. Writing $\Delta \Gamma^{(1)}=\int d \tau \Delta \mathcal{L}^{(1)}$, this is equivalent to

$$
\delta_{\epsilon}^{(0)} \Delta \mathcal{L}^{(1)}=\frac{d X}{d \tau}
$$

where $X$ is an arbitrary expression of order 3 . What we shall do is to generate the most general expressions for $\Delta \mathcal{L}^{(1)}$ and $X$ allowed by $S O(9)$ and discrete CPT symmetries and demonstrate that the only solution is $\Delta \mathcal{L}^{(1)}=0$ up to a total derivative, i.e. $\Delta \Gamma^{(1)}=0$.

It should be emphasized that our method actually does not rely on the detailed forms of $\Gamma^{(1)}$ and $\delta_{\epsilon}^{(1)}$. We may take this as a strong indication that this uniqueness property would persist to higher orders. Although restricted to the source-probe configuration, our

*The concept of "order" in the derivative expansion is defined, as usual, as the number of time derivatives plus half the number of fermions. For the importance of the derivative expansion scheme, see the detailed discussions presented in [1]. 
result reveals in a clear way the unusual power of maximal supersymmetry in controlling even the off-shell structures of Matrix theory.

The organization of the rest of the article is as follows: In Sec. 2, we give a brief description of Matrix theory and its "source-probe" configuration, to be studied in this article. It includes the explanation of how supersymmetry, $S O(9)$ symmetry and discrete C-P-T symmetries act in this setting. The analysis of the SUSY Ward identity is performed in Sec. 3. After elucidating our strategy, we describe how to generate the most general form of $\Delta \mathcal{L}^{(1)}$ and the possible total derivative terms in its SUSY transform and reduce them by the use of various Fierz identities to truly independent structures. Using the results of these analyses, the SUSY Ward identity (1.1) is transformed into sets of linear coupled equations for the coefficients of such structures. Upon solving them we shall find that $\Delta \mathcal{L}^{(1)}$ must identically vanish, demonstrating the uniqueness of the solution of the Ward identity at 1-loop at order 4. Sec. 4 is devoted to discussion of the implication of our result and some further remarks. In the Appendix, the Fierz identities used in the main text are collected.

\section{Preliminaries}

The classical action for the $U(N+1)$ Matrix theory in the Euclidean formulation is given by

$$
\begin{aligned}
S_{0} & =\int d \tau \operatorname{Tr}\left(\frac{1}{2}\left[D_{\tau}, X^{m}\right]^{2}-\frac{g^{2}}{4}\left(\left[X^{m}, X^{n}\right]\right)^{2}+\frac{1}{2} \Theta^{T}\left[D_{\tau}, \Theta\right]-\frac{g}{2} \Theta^{T} \gamma^{m}\left[X_{m}, \Theta\right]\right) \\
D_{\tau} & \equiv \partial_{\tau}-i g A
\end{aligned}
$$

In this expression, $X_{i j}^{m}(\tau), A_{i j}(\tau)$ and $\Theta_{\alpha, i j}(\tau)$ are the $(N+1) \times(N+1)$ hermitian matrix fields, representing the bosonic part of the D-particles, the gauge fields, and the fermionic part of the D-particles, respectively. $D_{\tau}$ is the covariant derivative, $\gamma^{m}$ are the real symmetric $16 \times 16 S O(9) \gamma$-matrices, and the vector index $m$ runs from 1 to 9 .

Among the number of important symmetries possessed by this action, our main focus will be the supersymmetry. It carries 16 spinorial parameters $\epsilon_{\alpha}$ and transforms the basic fields as

$$
\begin{aligned}
& \delta_{\epsilon} A=\epsilon^{T} \Theta, \quad \delta_{\epsilon} X^{m}=-i \epsilon^{T} \gamma^{m} \Theta \\
& \delta_{\epsilon} \Theta=i\left(\left[D_{\tau}, X_{m}\right] \gamma^{m}+\frac{g}{2}\left[X_{m}, X_{n}\right] \gamma^{m n}\right) \epsilon .
\end{aligned}
$$

Although the SUSY algebra closes only on-shell up to field-dependent gauge transforma- 
tions, $S_{0}$ is invariant under (2.3) and (2.4) أ.

For the present investigation, two additional symmetries play crucial roles. One is the $S O(9)$ symmetry. Besides giving restrictions on the forms of various quantities, it provides highly non-trivial relations among terms involving fermions through numerous Fierz identities. The other is the discrete C-P-T symmetries inherited from the 10-dimensional super Yang-Mills theory, from which the above action can be obtained by dimensional reduction. To understand these symmetries properly, tentatively we go back to the Minkowski formulation. Since the fermions in Matrix theory are Weyl-projected (as well as Mojorana), $\mathrm{P}$ and $\mathrm{T}$ are separately violated while $\mathrm{C}$ and $\mathrm{CPT}$ symmetries remain preserved. Under the C-transformation, the Minkowski fields transform as

$$
\Theta_{\alpha} \rightarrow \Theta_{\alpha}^{T}, \quad A_{0} \rightarrow-A_{0}^{T}, \quad X_{m} \rightarrow-X_{m}^{T}
$$

where the superscript $T$ denotes the matrix-transposition. On the other hand, one finds that the CPT-transformation does not transform the fields but flips the sign of the timederivative and effects $i \rightarrow-i$ as it is anti-unitary.

Now let us give a brief description of the "source-probe" situation, on which our investigation will be focused. It is the configuration of a (probe) D-particle interacting with a large number, $N$, of (source) D-particles all sitting at the origin. This is expressed by the splitting

$$
\begin{array}{rlrl}
X^{m}(\tau) & =\frac{1}{g} B^{m}(\tau)+Y^{m}(\tau), & \Theta^{\alpha}(\tau)=\frac{1}{g} \theta^{\alpha}(\tau)+\Psi^{\alpha}(\tau), \\
B^{m}(\tau)=\operatorname{diag}(r^{m}(\tau), \underbrace{0, \cdots, 0}_{N}), & \theta^{\alpha}(\tau)=\operatorname{diag}(\theta^{\alpha}(\tau), \underbrace{0, \cdots, 0}_{N}),
\end{array}
$$

where $B_{m}(\tau)$ and $\theta_{\alpha}(\tau)$ are the bosonic and the fermionic backgrounds expressing the positions and the spin degrees of freedom of the D-particles respectively and $Y_{m}(\tau)$ and $\Psi_{\alpha}(\tau)$ denote the quantum fluctuations around them. We will be interested in the fully off-shell effective action $\Gamma\left[r^{m}, \theta_{\alpha}\right]$ of the probe D-particle after all the fluctuations are integrated out. In other words, we shall not impose any equations of motion for $r_{m}(\tau)$ and $\theta_{\alpha}(\tau)$, i.e. they will be arbitrary functions of $\tau$. At the tree level, the effective action and the SUSY transformations take the form

$$
\begin{aligned}
\widetilde{\Gamma}^{(0)} & =\int d \tau\left(\frac{v^{2}}{2 g^{2}}+\frac{\theta \dot{\theta}}{2 g^{2}}\right), \\
\delta_{\epsilon}^{(0)} r^{m} & =-i \epsilon \gamma^{m} \theta, \quad \delta_{\epsilon}^{(0)} \theta_{\alpha}=i(\psi \epsilon)_{\alpha} .
\end{aligned}
$$

\footnotetext{
†Strictly speaking, this symmetry is not supersymmetry since the bose and fermi dergrees of freedom do not match off-shell; it is a symmetry that becomes SUSY on-shell. Nevertheless, in this article we shall call (2.3) and (2.4) SUSY transformations, following common usage.
} 
Here and hereafter, the dot means differentiation with respect to the Euclidean time $\tau$ and we will use $v^{m}$ and $a^{m}$ to denote $\dot{r}^{m}$ and $\ddot{r}^{m}$ respectively. Contractions of the spinor indices are often suppressed, so that $\theta \dot{\theta}$ stands for $\theta_{\alpha} \dot{\theta}_{\alpha}$, etc.

For the source-probe configuration described above, the restrictions imposed on the effective action by the $\mathrm{C}$ and CPT symmetries, the $S O(9)$ symmetry and the hermiticity requirement can be worked out in the following way. Again to avoid misunderstanding we must first go back to the Minkowski formulation, in which the time $t$ is related to the Euclidean time $\tau$ by $t=-i \tau$, and then translate the results into the Euclidean language.

1. The C-transformation only affects $r^{m}$ as $r^{m} \rightarrow-r^{m}$ and dictates that the number of $r^{m}$ 's must be even.

2. The $S O(9)$ invariance imposes two types of restrictions. First, the spinors must come in bilinears so that the spinor indices are contracted. Next, since the vector indices must also be contracted to produce $S O(9)$ singlets, the number of such indices must be even. This together with the C-invariance above dictates that the total number of $\gamma^{m}$ 's must be even.

3. Let $\theta^{(m)}$ denote the $m$-th $t$-derivative of $\theta$. Then, any fermion bilinear of the form $\theta^{(m)} A \theta^{(n)}$ with $A$ a real matrix (such as $\left.\gamma^{i_{1} i_{2} \ldots i_{k}}\right)$ is anti-hermitian since $\left(\theta^{(m)} A \theta^{(n)}\right)^{\dagger}=$ $\theta^{(n)} A^{T} \theta^{(m)}=-\theta^{(m)} A \theta^{(n)}$. Thus, a hermitian spinor bilinear, to be used as a building block, must be of the form $i \theta^{(m)} A \theta^{(n)}$, i.e. with an overall $i$.

4. Now consider the CPT transformation. On the bosonic coordinates, its only effect is to change the sign of the time derivative and hence we have $r^{m} \rightarrow r^{m}, v^{m} \rightarrow-v^{m}$, etc. This is often referred to as "time-reversal" from the M-theory point of view. It gives the well known restriction that the number of time-derivatives in the purely bosonic terms must be even. On the other hand, due to an overall $i$, CPT transform of a hermitian fermion bilinear $i \theta^{(m)} A \theta^{(n)}$ acquires an extra minus sign from $i \rightarrow-i$, apart from the factors of -1 from the time-derivatives. This means that we can count $\theta^{2}$ as one time-derivative as far as the CPT invariance is concerned. Combined with the requirement for the bosonic part, this amounts to a remarkably simple statement that CPT invariance is equivalent to demanding the "order", as defined in the sense of the derivative expansion, to be even.

Going back to the Euclidean formulation by $t \rightarrow-i \tau$, we can summarize the above requirements as follows: 
- In constructing the effective Lagrangian, use $i^{1+m+n} \theta^{(m)} \gamma^{i_{1} i_{2} \ldots i_{k}} \theta^{(n)}$ as fermionic building block and demand that the number of $r^{m}$, the number of $\gamma^{m}$ and the "order" be all even.

This rule will facilitate the generation of the most general effective action, to be performed in the next section.

\section{Analysis of the SUSY Ward identity}

\section{$3.1 \quad$ Strategy}

We begin the analysis of the SUSY Ward identity by explaining our strategy. As was already explained in the introduction, in terms of the unintegrated quantities the Ward identity we wish to analyze is

$$
\delta_{\epsilon}^{(0)} \Delta \mathcal{L}^{(1)}=\frac{d X}{d \tau}
$$

where $\Delta \mathcal{L}^{(1)}$ is the most general effective Lagrangian possible at 1-loop at order 4 and $X$ stands for an arbitrary expression at order 3 . To solve this equation, we expand $\Delta \mathcal{L}^{(1)}$ and $X$ in powers of $\theta$ in the manner

$$
\begin{aligned}
\Delta \mathcal{L}^{(1)} & \equiv \Delta \mathcal{L}_{\theta^{0}}^{(1)}+\Delta \mathcal{L}_{\theta^{2}}^{(1)}+\Delta \mathcal{L}_{\theta^{4}}^{(1)}+\Delta \mathcal{L}_{\theta^{6}}^{(1)}+\Delta \mathcal{L}_{\theta^{8}}^{(1)} \\
X & \equiv X_{\epsilon \partial^{3} \theta}+X_{\epsilon \partial^{2} \theta^{3}}+X_{\epsilon \partial \theta^{5}}+X_{\epsilon \theta^{7}}
\end{aligned}
$$

The subscripts indicate the structure of each term schematically and should be selfexplanatory. Now the tree-level SUSY variation $\delta_{\epsilon}^{(0)}$ consists of $\delta_{r}^{(0)}$ which acts on $r^{m}$ to add one $\theta$ and $\delta_{\theta}^{(0)}$ that acts on $\theta_{\alpha}$ to remove one $\theta$. Thus, by substituting the expansions (3.2) and (3.3) into the equation (3.1) and collecting terms with the same number of $\theta$ 's, the Ward identity can be split into the following five equations:

$$
\begin{aligned}
& \delta_{r}^{(0)} \Delta \mathcal{L}_{\theta^{0}}^{(1)}+\delta_{\theta}^{(0)} \Delta \mathcal{L}_{\theta^{2}}^{(1)}=\frac{d X_{\epsilon \partial^{3} \theta}}{d \tau}, \\
& \delta_{r}^{(0)} \Delta \mathcal{L}_{\theta^{2}}^{(1)}+\delta_{\theta}^{(0)} \Delta \mathcal{L}_{\theta^{4}}^{(1)}=\frac{d X_{\epsilon \partial^{2} \theta^{3}}}{d \tau}, \\
& \delta_{r}^{(0)} \Delta \mathcal{L}_{\theta^{4}}^{(1)}+\delta_{\theta}^{(0)} \Delta \mathcal{L}_{\theta^{6}}^{(1)}=\frac{d X_{\epsilon \partial \theta^{5}}}{d \tau} \\
& \delta_{r}^{(0)} \Delta \mathcal{L}_{\theta^{6}}^{(1)}+\delta_{\theta}^{(0)} \Delta \mathcal{L}_{\theta^{8}}^{(1)}=\frac{d X_{\epsilon \theta^{7}}}{d \tau}, \\
& \delta_{r}^{(0)} \Delta \mathcal{L}_{\theta^{8}}^{(1)}=0 .
\end{aligned}
$$


Our task then is to solve each of these equations to determine $\Delta \mathcal{L}_{\theta^{n}}^{(1)}$. Since both $\Delta \mathcal{L}_{\theta^{n}}$ 's and $X_{\epsilon \partial^{n} \theta^{m}}$ 's are unknown, except for restrictions imposed by $S O(9)$ and CPT symmetries, one might at first sight suspect that the solution would not be unique. As we shall see, this expectation will be refuted.

\subsection{General form of the effective Lagrangian}

We will generate the most general expressions for $\Delta \mathcal{L}_{\theta^{n}}^{(1)}$ 's in following steps: First, we enumerate all possible structures contributing to $\Delta \mathcal{L}_{\theta^{n}}^{(1)}$ at order 4 , taking into account the symmetry requirement spelled out at the end of the previous section. Next we will eliminate all possible total derivative combinations. Finally, for terms containing $4 \theta$ 's or more, we apply appropriate $S O(9)$ Fierz identities to reduce the expressions to truly independent ones.

Let us describe this procedure for each $\Delta \mathcal{L}_{\theta^{n}}^{(1)}$ more explicitly. In the following, $A_{i} \sim E_{i}$ will denote arbitrary constants.

Expression for $\Delta \mathcal{L}_{\theta^{0}}^{(1)}$ :

For the purely bosonic part, it is straightforward to see that there are 6 independent terms, which can be chosen to be

$$
\Delta \mathcal{L}_{\theta^{0}}^{(1)}=\frac{A_{1}(r \cdot v)^{4}}{r^{11}}+\frac{A_{2}(r \cdot a)^{2}}{r^{7}}+\frac{A_{3} v^{2}(r \cdot v)^{2}}{r^{9}}+\frac{A_{4} v^{2}(r \cdot a)}{r^{7}}+\frac{A_{5} v^{4}}{r^{7}}+\frac{A_{6} a^{2}}{r^{5}} .
$$

Expression for $\Delta \mathcal{L}_{\theta^{2}}^{(1)}$ :

Similarly, it is not difficult to enumerate all possible terms with two $\theta$ 's and we find the following 11 independent terms:

$$
\begin{aligned}
\Delta \mathcal{L}_{\theta^{2}}^{(1)}= & +\frac{B_{1}(\ddot{\theta} \dot{\theta})}{r^{5}}+\frac{B_{2}(r \cdot v)^{2}(\dot{\theta} \theta)}{r^{9}}+\frac{B_{3}(r \cdot a)(\dot{\theta} \theta)}{r^{7}}+\frac{B_{4} v^{2}(\dot{\theta} \theta)}{r^{7}}+\frac{B_{5} r_{j} v_{i}\left(\dot{\theta} \gamma^{i j} \dot{\theta}\right)}{r^{7}} \\
& +\frac{B_{6} r_{j} v_{i}(r \cdot v)\left(\dot{\theta} \gamma^{i j} \theta\right)}{r^{9}}+\frac{B_{7}(r \cdot v)^{2} r_{j} v_{i}\left(\theta \gamma^{i j} \theta\right)}{r^{11}}+\frac{B_{8} v^{2} r_{j} v_{i}\left(\theta \gamma^{i j} \theta\right)}{r^{9}} \\
& +\frac{B_{9} r_{j} a_{i}\left(\dot{\theta} \gamma^{i j} \theta\right)}{r^{7}}+\frac{B_{10} r_{j} a_{i}(r \cdot v)\left(\theta \gamma^{i j} \theta\right)}{r^{9}}+\frac{B_{11} v_{i} a_{j}\left(\theta \gamma^{i j} \theta\right)}{r^{7}}
\end{aligned}
$$

Expression for $\Delta \mathcal{L}_{\theta^{4}}^{(1)}$

Beginning at this order with $4 \theta$ 's, our task becomes much more difficult. In addition to the number of allowed terms getting large, we must find judicious Fierz identities to reduce them to independent expressions. Since these identities are rather involved, 
we shall relegate them to Appendix. They are generated by an efficient new algorithm developed in the previous work[2].

After eliminating terms related by total derivatives, the ones contributing to $\Delta \mathcal{L}_{\theta^{4}}^{(1)}$ are found to be

$$
\begin{aligned}
& \frac{\left(\theta \gamma^{a_{1} a_{2}} \theta\right)\left(\dot{\theta} \gamma^{a_{1} a_{2}} \dot{\theta}\right)}{r^{7}}, \frac{\left(\theta \gamma^{a_{1} a_{2} a_{3}} \theta\right)\left(\dot{\theta} \gamma^{a_{1} a_{2} a_{3}} \dot{\theta}\right)}{r^{7}}, \frac{(r \cdot v)\left(\theta \gamma^{a_{1} a_{2}} \theta\right)\left(\dot{\theta} \gamma^{a_{1} a_{2}} \theta\right)}{r^{9}}, \\
& \frac{(r \cdot v)\left(\theta \gamma^{a_{1} a_{2} a_{3}} \theta\right)\left(\dot{\theta} \gamma^{a_{1} a_{2} a_{3}} \theta\right)}{r^{9}}, \frac{(r \cdot v)^{2}\left(\theta \gamma^{a_{1} a_{2}} \theta\right)^{2}}{r^{11}}, \frac{(r \cdot v)^{2}\left(\theta \gamma^{a_{1} a_{2} a_{3}} \theta\right)^{2}}{r^{11}}, \\
& \frac{r_{i_{1}} r_{i_{2}}(r \cdot v)^{2}\left(\theta \gamma^{a_{1} i_{1}} \theta\right)\left(\theta \gamma^{a_{1} i_{2}} \theta\right)}{r^{13}}, \frac{r_{i_{1}} r_{i_{2}}\left(\theta \gamma^{a_{1} i_{2}} \theta\right)\left(\dot{\theta} \gamma^{a_{1} i_{1}} \dot{\theta}\right)}{r^{9}}, \frac{r_{i_{1}} r_{i_{2}}(r \cdot v)\left(\theta \gamma^{a_{1} i_{2}} \theta\right)\left(\dot{\theta} \gamma^{a_{1} i_{1}} \theta\right)}{r^{11}}, \\
& \frac{r_{i_{1}} r_{i_{2}}(r \cdot v)^{2}\left(\theta \gamma^{a_{1} a_{2} i_{1}} \theta\right)\left(\theta \gamma^{a_{1} a_{2} i_{2}} \theta\right)}{r^{13}}, \frac{r_{i_{1}} r_{i_{2}}\left(\theta \gamma^{a_{1} a_{2} i_{2}} \theta\right)\left(\dot{\theta} \gamma^{a_{1} a_{2} i_{1}} \dot{\theta}\right)}{r^{9}}, \frac{v^{2}\left(\theta \gamma^{a_{1} a_{2}} \theta\right)^{2}}{r^{9}}, \\
& \frac{v^{2}\left(\theta \gamma^{a_{1} a_{2} a_{3}} \theta\right)^{2}}{r^{9}}, \frac{r_{i_{1}} r_{i_{2}}(r \cdot v)\left(\theta \gamma^{a_{1} a_{2} i_{2}} \theta\right)\left(\dot{\theta} \gamma^{a_{1} a_{2} i_{1}} \theta\right)}{r^{11}}, \frac{v^{2} r_{i_{1}} r_{i_{2}}\left(\theta \gamma^{a_{1} i_{1}} \theta\right)\left(\theta \gamma^{a_{1} i_{2}} \theta\right)}{r^{11}}, \\
& \frac{v^{2} r_{i_{1}} r_{i_{2}}\left(\theta \gamma^{a_{1} a_{2} i_{1}} \theta\right)\left(\theta \gamma^{a_{1} a_{2} i_{2}} \theta\right)}{r^{11}}, \frac{r_{i_{1}} v_{i_{2}}(\dot{\theta} \theta)\left(\theta \gamma^{i_{1} i_{2}} \theta\right)}{r^{9}}, \frac{r_{i_{1}} v_{i_{2}}(r \cdot v)\left(\theta \gamma^{a_{1} i_{1}} \theta\right)\left(\theta \gamma^{a_{1} i_{2}} \theta\right)}{r^{11}}, \\
& \frac{r_{i_{1}} v_{i_{2}}(r \cdot v)\left(\theta \gamma^{a_{1} a_{2} i_{1}} \theta\right)\left(\theta \gamma^{a_{1} a_{2} i_{2}} \theta\right)}{r^{11}}, \frac{r_{i_{1}} v_{i_{2}}\left(\theta \gamma^{a_{1} i_{1} i_{2}} \theta\right)\left(\dot{\theta} \gamma^{a_{1}} \theta\right)}{r^{9}}, \frac{r_{i_{1}} v_{i_{2}}\left(\theta \gamma^{a_{1} i_{1}} \theta\right)\left(\dot{\theta} \gamma^{a_{1} i_{2}} \theta\right)}{r^{9}} \\
& \frac{r_{i_{1}} v_{i_{2}}\left(\theta \gamma^{a_{1} i_{2}} \theta\right)\left(\dot{\theta} \gamma^{a_{1} i_{1}} \theta\right)}{r^{9}}, \frac{r_{i_{1}} v_{i_{2}}\left(\theta \gamma^{a_{1} a_{2} i_{1}} \theta\right)\left(\dot{\theta} \gamma^{a_{1} a_{2} i_{2}} \theta\right)}{r^{9}}, \frac{r_{i_{1}} v_{i_{2}}\left(\theta \gamma^{a_{1} a_{2}} \theta\right)\left(\dot{\theta} \gamma^{a_{1} a_{2} i_{1} i_{2}} \theta\right)}{r^{9}}, \\
& \frac{v_{i_{1}} v_{i_{2}}\left(\theta \gamma^{a_{1} i_{1}} \theta\right)\left(\theta \gamma^{a_{1} i_{2}} \theta\right)}{r^{9}}, \frac{r_{i_{1}} v_{i_{2}}\left(\theta \gamma^{a_{1} a_{2} i_{2}} \theta\right)\left(\dot{\theta} \gamma^{a_{1} a_{2} i_{1}} \theta\right)}{r^{9}}, \frac{v_{i_{1}} v_{i_{2}}\left(\theta \gamma^{a_{1} a_{2} i_{1}} \theta\right)\left(\theta \gamma^{a_{1} a_{2} i_{2}} \theta\right)}{r^{9}}, \\
& \frac{r_{i_{1}} r_{i_{2}} v_{i_{3}} v_{i_{4}}\left(\theta \gamma^{a_{1} i_{1} i_{3}} \theta\right)\left(\theta \gamma^{a_{1} i_{2} i_{4}} \theta\right)}{r^{11}}, \frac{r_{i_{1}} r_{i_{2}} v_{i_{3}} v_{i_{4}}\left(\theta \gamma^{i_{1} i_{3}} \theta\right)\left(\theta \gamma^{i_{2} i_{4}} \theta\right)}{r^{11}} \\
& \frac{r_{i_{1}} v_{i_{2}}\left(\theta \gamma^{a_{1} a_{2} a_{3}} \theta\right)\left(\dot{\theta} \gamma^{a_{4} a_{5} a_{6} a_{7}} \theta\right) \epsilon_{a_{1} a_{2} a_{3} a_{4} a_{5} a_{6} a_{7} i_{1} i_{2}}}{r^{9}} .
\end{aligned}
$$

There are altogether 30 terms, which are not yet all independent. We now indicate how they can be reduced to the independent ones by the use of Fierz identities.

First, several terms in the above list simply vanish due to the Fierz identities (A.1) and (A.2). Next, the last term, the only term with the $\epsilon$-tensor, can be rewritten into an expression without it by using the identity (A.3). It becomes

$$
\frac{r_{i_{1}} v_{i_{2}}\left(\theta \gamma^{a_{1} a_{2} a_{3}} \theta\right)\left(\dot{\theta} \gamma^{a_{4} a_{5} a_{6} a_{7}} \theta\right) \epsilon_{a_{1} a_{2} a_{3} a_{4} a_{5} a_{6} a_{7} i_{1} i_{2}}}{r^{9}}=720 \frac{r_{i_{1}} v_{i_{2}}(\dot{\theta} \theta)\left(\theta \gamma^{i_{1} i_{2}} \theta\right)}{r^{9}} .
$$

Similarly, we can relate various other terms by using the identities (A.4) (A.10).

After all possible such manipulations, the number of independent structures is reduced 
to 13 and we get

$$
\begin{aligned}
\Delta \mathcal{L}_{\theta^{4}}^{(1)}= & \frac{C_{1}(\dot{\theta} \theta)^{2}}{r^{7}}+\frac{C_{2}\left(\dot{\theta} \gamma^{i} \theta\right)^{2}}{r^{7}}+\frac{C_{3} r_{i} r_{j}\left(\dot{\theta} \gamma^{i} \theta\right)\left(\dot{\theta} \gamma^{j} \theta\right)}{r^{9}}+\frac{C_{4} r_{j} r_{k}\left(\theta \gamma^{i j} \theta\right)\left(\dot{\theta} \gamma^{i k} \dot{\theta}\right)}{r^{9}} \\
& +\frac{C_{5} r_{j} r_{k}(r \cdot v)\left(\theta \gamma^{i j} \theta\right)\left(\dot{\theta} \gamma^{i k} \theta\right)}{r^{11}}+\frac{C_{6} r_{j} r_{k}(r \cdot v)^{2}\left(\theta \gamma^{i j} \theta\right)\left(\theta \gamma^{i k} \theta\right)}{r^{13}} \\
& +\frac{C_{7} r_{j} r_{k} v^{2}\left(\theta \gamma^{i j} \theta\right)\left(\theta \gamma^{i k} \theta\right)}{r^{11}}+\frac{C_{8} r_{i} v_{j}(\dot{\theta} \theta)\left(\theta \gamma^{i j} \theta\right)}{r^{9}}+\frac{C_{9} r_{j} v_{k}\left(\theta \gamma^{i k} \theta\right)\left(\dot{\theta} \gamma^{i j} \theta\right)}{r^{9}} \\
& +\frac{C_{10} r_{j} v_{k}\left(\theta \gamma^{i j} \theta\right)\left(\dot{\theta} \gamma^{i k} \theta\right)}{r^{9}}+\frac{C_{11} r_{j} v_{k}(r \cdot v)\left(\theta \gamma^{i j} \theta\right)\left(\theta \gamma^{i k} \theta\right)}{r^{11}} \\
& +\frac{C_{12} v_{j} v_{k}\left(\theta \gamma^{i j} \theta\right)\left(\theta \gamma^{i k} \theta\right)}{r^{9}}+\frac{C_{13} r_{i} r_{k} v_{j} v_{l}\left(\theta \gamma^{i j} \theta\right)\left(\theta \gamma^{k l} \theta\right)}{r^{11}}
\end{aligned}
$$

Expression for $\Delta \mathcal{L}_{\theta^{6}}^{(1)}$

Now we come to the structures with $6 \theta$ 's. The number of possible terms, disregarding total derivatives, turned out to be 45. As they are too space-filling to be displayed here, we shall only sketch the reduction steps.

First, a number of terms containing an $\epsilon$-tensor can all be turned into those without one by the identities $(\mathrm{A} .11) \sim(\mathrm{A} .15)$ and $(\mathrm{A.3})$. The remaining terms can then be reduced by using the identities $\left(\right.$ A.16) $\sim\left(\right.$ A.18) as well as those already used at $\mathcal{O}\left(\theta^{4}\right)$.

These manipulations drastically reduce the number of independent terms and we end up with the following simple expression:

$$
\begin{aligned}
\Delta \mathcal{L}_{\theta^{6}}^{(1)}= & \frac{D_{1} r_{j} r_{k}(\dot{\theta} \theta)\left(\theta \gamma^{i j} \theta\right)\left(\theta \gamma^{i k} \theta\right)}{r^{11}}+\frac{D_{2} r_{k} r_{l}\left(\theta \gamma^{i j} \theta\right)\left(\theta \gamma^{i k} \theta\right)\left(\dot{\theta} \gamma^{j l} \theta\right)}{r^{11}} \\
& +\frac{D_{3} r_{l} v_{k}\left(\theta \gamma^{i j} \theta\right)\left(\theta \gamma^{i k} \theta\right)\left(\theta \gamma^{j l} \theta\right)}{r^{11}}+\frac{D_{4} r_{j} r_{k} r_{l} v_{m}\left(\theta \gamma^{i j} \theta\right)\left(\theta \gamma^{i k} \theta\right)\left(\theta \gamma^{l m} \theta\right)}{r^{13}}
\end{aligned}
$$

Expression for $\Delta \mathcal{L}_{\theta^{8}}^{(1)}$ :

Finally, we are left with the expression with $8 \theta^{\prime}$ 's, the maximum number allowed at order 4. Starting from 36 possible such expressions, application of appropriate Fierz identities listed in the Appendix leads to

$$
\begin{aligned}
\Delta \mathcal{L}_{\theta^{8}}^{(1)}= & \frac{E_{1}\left(\theta \gamma^{i j} \theta\right)\left(\theta \gamma^{i k} \theta\right)\left(\theta \gamma^{j l} \theta\right)\left(\theta \gamma^{k l} \theta\right)}{r^{11}}+\frac{E_{2} r_{k} r_{m}\left(\theta \gamma^{i j} \theta\right)\left(\theta \gamma^{i k} \theta\right)\left(\theta \gamma^{j l} \theta\right)\left(\theta \gamma^{l m} \theta\right)}{r^{13}} \\
& +\frac{E_{3} r_{j} r_{k} r_{m} r_{n}\left(\theta \gamma^{i j} \theta\right)\left(\theta \gamma^{i k} \theta\right)\left(\theta \gamma^{l m} \theta\right)\left(\theta \gamma^{l n} \theta\right)}{r^{15}}
\end{aligned}
$$

This agrees with the result of [9, 13], where the effective action without time-derivatives of $\theta$ was considered. 
This completes the construction of the most general effective Lagrangian. We now turn to the generation of allowed expression for the quantity $X$ at order 3, from which we produce the total derivative term $d X / d \tau$ in the basic Ward identity (3.1).

\subsection{Generation of total derivative terms}

The basic steps for generating the general order 3 expressions $X_{\epsilon \partial^{m} \theta^{n}}$ are quite similar to the ones for $\Delta \mathcal{L}_{\theta^{n}}^{(1)}$ described in the previous section. Fortunately, we can argue that of the four such $X_{\epsilon \partial^{m} \theta^{n}}$ 's two of them, namely $X_{\epsilon \partial^{3} \theta}$ and $X_{\epsilon \partial \theta^{5}}$, can be set to zero.

First, consider the terms of $\mathcal{O}\left(\epsilon \partial^{4} \theta\right)$ generated on the left-hand side (LHS) of (3.4). Schematically, they can be grouped into the following 5 types:

$$
\begin{aligned}
& Y_{1}[r]\left(\epsilon \gamma^{*} \theta^{(4)}\right), \quad Y_{2}[r, v]\left(\epsilon \gamma^{*} \theta^{(3)}\right), \quad Y_{3}[r, v, a]\left(\epsilon \gamma^{*} \ddot{\theta}\right), \\
& Y_{4}[r, v, a, \dot{a}]\left(\epsilon \gamma^{*} \dot{\theta}\right), \quad Y_{5}[r, v, a, \dot{a}, \ddot{a}]\left(\epsilon \gamma^{*} \theta\right),
\end{aligned}
$$

where $Y_{1} \sim Y_{5}$ are functions of $r(\tau)$ and its derivatives and $*$ in $\gamma^{*}$ stands for various sets of vector indices. Since total derivatives can be absorbed into $d X_{\epsilon \partial^{3} \theta} / d \tau$ on the righthand side (RHS), the first 4 structures can be transformed into the last one in the list by "integration by parts". This process is unambiguous since there is only one $\theta$ involved and we may simply transfer the derivatives on it to bosonic variables. Thus, Eq. (3.4) is simplified to

$$
Y_{5}[r, v, a, \dot{a}, \ddot{a}]\left(\epsilon \gamma^{*} \theta\right)=\frac{d X_{\epsilon \partial^{3} \theta}}{d \tau}
$$

Now note that while the LHS has no derivatives of $\theta, d / d \tau$ on the RHS necessarily produces $\dot{\theta}$ for each term composing $X_{\epsilon \partial^{3} \theta}$. This is a contradiction unless $X_{\epsilon \partial^{3} \theta}$ vanishes.

Similar argument applies to $\mathcal{O}\left(\epsilon \partial^{2} \theta^{5}\right)$ terms in the Ward identity (3.6). In this case, the structure of $X_{\epsilon \partial \theta^{5}}$ is schematically

$$
X_{\epsilon \partial \theta^{5}} \sim Z_{1}[r] \epsilon \theta^{4} \dot{\theta}+Z_{2}[r] \epsilon \theta^{5} \dot{r}
$$

where $Z_{1}[r]$ and $Z_{2}[r]$ are some functions of $r(\tau)$. The time derivative of this expression produces either $\ddot{r}^{n}$ or $\ddot{\theta}^{\alpha}$. However, the LHS of (3.6) can be transformed by integration by parts to expressions without such double derivatives. Hence we may set $X_{\epsilon \partial \theta^{5}}=0$.

For the remaining two entities $X_{\epsilon \partial^{2} \theta^{3}}$ and $X_{\epsilon \theta^{7}}$, no such arguments apply and we must generate all possible terms which are algebraically independent. 
Construction of $X_{\epsilon \partial^{2} \theta^{3}}$ : There are 8 possible types of structures, up to total derivatives:

$$
\begin{aligned}
& \text { type } 1:\left(\epsilon \gamma^{*} \theta\right)\left(\theta \gamma^{*} \theta\right) \ddot{r}^{n}, \\
& \text { type } 2:\left(\epsilon \gamma^{*} \theta\right)\left(\theta \gamma^{*} \theta\right) \dot{r}^{n} \dot{r}^{m}, \\
& \text { type } 3:\left(\epsilon \gamma^{*} \dot{\theta}\right)\left(\theta \gamma^{*} \theta\right) \dot{r}^{n}, \quad \text { type } 3^{\prime}:\left(\epsilon \gamma^{*} \theta\right)\left(\theta \gamma^{*} \dot{\theta}\right) \dot{r}^{n}, \\
& \text { type } 4:\left(\epsilon \gamma^{*} \ddot{\theta}\right)\left(\theta \gamma^{*} \theta\right) r^{n}, \quad \text { type } 4^{\prime}:\left(\epsilon \gamma^{*} \theta\right)\left(\ddot{\theta} \gamma^{*} \theta\right) r^{n}, \\
& \text { type } 5:\left(\epsilon \gamma^{*} \theta\right)\left(\dot{\theta} \gamma^{*} \dot{\theta}\right) r^{n}, \quad \text { type } 5^{\prime}:\left(\epsilon \gamma^{*} \dot{\theta}\right)\left(\theta \gamma^{*} \dot{\theta}\right) r^{n} .
\end{aligned}
$$

Structures of type $3^{\prime}$ can be expressed in terms of those of type 3 by the use of the Fierz identities of the type $\left(\epsilon \gamma^{*} \theta\right)\left(\theta \gamma^{*} \dot{\theta}\right) \rightarrow\left(\epsilon \gamma^{*} \dot{\theta}\right)\left(\theta \gamma^{*} \theta\right)$. Similarly, type $4^{\prime}$ and type $5^{\prime}$ terms can be rewritten in terms of type 4 and type 5 terms respectively. Thus we only need to consider the structures of type $1 \sim$ type 5 .

Possible type 1 terms are given by

$\frac{r_{i_{1}}(r \cdot a)\left(\epsilon \gamma^{a_{1} a_{2} i_{1}} \theta\right)\left(\theta \gamma^{a_{1} a_{2}} \theta\right)}{r^{9}}, \frac{a_{i_{1}}\left(\epsilon \gamma^{a_{1} a_{2} i_{1}} \theta\right)\left(\theta \gamma^{a_{1} a_{2}} \theta\right)}{r^{7}}, \frac{r_{i_{1}}(r \cdot a)\left(\epsilon \gamma^{a_{1}} \theta\right)\left(\theta \gamma^{a_{1} i_{1}} \theta\right)}{r^{9}}$,

$\frac{r_{i_{1}} r_{i_{2}} a_{i_{3}}\left(\epsilon \gamma^{a_{1} i_{1} i_{3}} \theta\right)\left(\theta \gamma^{a_{1} i_{2}} \theta\right)}{r^{9}}, \frac{a_{i_{1}}\left(\epsilon \gamma^{a_{1}} \theta\right)\left(\theta \gamma^{a_{1} i_{1}} \theta\right)}{r^{7}}, \frac{r_{i_{1}} r_{i_{2}} a_{i_{3}}\left(\epsilon \gamma^{i_{1}} \theta\right)\left(\theta \gamma^{i_{2} i_{3}} \theta\right)}{r^{9}}$,

$\frac{r_{i_{1}}(r \cdot a)\left(\epsilon \gamma^{a_{1} a_{2} a_{3} i_{1}} \theta\right)\left(\theta \gamma^{a_{1} a_{2} a_{3}} \theta\right)}{r^{9}}, \frac{a_{i_{1}}\left(\epsilon \gamma^{a_{1} a_{2} a_{3} i_{1}} \theta\right)\left(\theta \gamma^{a_{1} a_{2} a_{3}} \theta\right)}{r^{7}}, \frac{r_{i_{1}}(r \cdot a)\left(\epsilon \gamma^{a_{1} a_{2}} \theta\right)\left(\theta \gamma^{a_{1} a_{2} i_{1}} \theta\right)}{r^{9}}$,

$\frac{r_{i_{1}} r_{i_{2}} a_{i_{3}}\left(\epsilon \gamma^{a_{1} a_{2} i_{1} i_{3}} \theta\right)\left(\theta \gamma^{a_{1} a_{2} i_{2}} \theta\right)}{r^{9}}, \frac{a_{i_{1}}\left(\epsilon \gamma^{a_{1} a_{2}} \theta\right)\left(\theta \gamma^{a_{1} a_{2} i_{1}} \theta\right)}{r^{7}}, \frac{r_{i_{1}} r_{i_{2}} a_{i_{3}}\left(\epsilon \gamma^{a_{1} i_{1}} \theta\right)\left(\theta \gamma^{a_{1} i_{2} i_{3}} \theta\right)}{r^{9}}$.

By applying the Fierz identities $(\mathrm{A.19}) \sim(\widehat{\mathrm{A} .23})$, they can be reduced to 5 independent structures of the form

$$
\begin{aligned}
& \frac{r_{i_{1}}(r \cdot a)\left(\epsilon \gamma^{a_{1}} \theta\right)\left(\theta \gamma^{a_{1} i_{1}} \theta\right)}{r^{9}}, \frac{a_{i_{1}}\left(\epsilon \gamma^{a_{1}} \theta\right)\left(\theta \gamma^{a_{1} i_{1}} \theta\right)}{r^{7}}, \frac{r_{i_{1}} r_{i_{2}} a_{i_{3}}\left(\epsilon \gamma^{a_{1} i_{1} i_{3}} \theta\right)\left(\theta \gamma^{a_{1} i_{2}} \theta\right)}{r^{9}}, \\
& \frac{r_{i_{1}} r_{i_{2}} a_{i_{3}}\left(\epsilon \gamma^{i_{1}} \theta\right)\left(\theta \gamma^{i_{2} i_{3}} \theta\right)}{r^{9}}, \frac{r_{i_{1}} r_{i_{2}} a_{i_{3}}\left(\epsilon \gamma^{a_{1} i_{1}} \theta\right)\left(\theta \gamma^{a_{1} i_{2} i_{3}} \theta\right)}{r^{9}} .
\end{aligned}
$$

In a similar fashion, we can write down all possible independent terms of type $2 \sim$ type 5 . The details are omitted.

Assembling all five types of structures, we obtain $X_{\epsilon \partial^{2} \theta^{3}}$ consisting of 31 independent 
terms with unknown coefficients $F_{i}$ :

$$
\begin{aligned}
& X_{\epsilon \partial^{2} \theta^{3}}=\frac{F_{1} r_{i_{1}}(r \cdot a)\left(\epsilon \gamma^{a_{1}} \theta\right)\left(\theta \gamma^{a_{1} i_{1}} \theta\right)}{r^{9}}+\frac{F_{2} a_{i_{1}}\left(\epsilon \gamma^{a_{1}} \theta\right)\left(\theta \gamma^{a_{1} i_{1}} \theta\right)}{r^{7}}+\frac{F_{3} r_{i_{1}} r_{i_{2}} a_{i_{3}}\left(\epsilon \gamma^{a_{1} i_{1} i_{3}} \theta\right)\left(\theta \gamma^{a_{1} i_{2}} \theta\right)}{r^{9}} \\
& +\frac{F_{4} r_{i_{1}} r_{i_{2}} a_{i_{3}}\left(\epsilon \gamma^{i_{1}} \theta\right)\left(\theta \gamma^{i_{2} i_{3}} \theta\right)}{r^{9}}+\frac{F_{5} r_{i_{1}}(r \cdot v)^{2}\left(\epsilon \gamma^{a_{1}} \theta\right)\left(\theta \gamma^{a_{1} i_{1}} \theta\right)}{r^{11}}+\frac{v^{2} F_{6} r_{i_{1}}\left(\epsilon \gamma^{a_{1}} \theta\right)\left(\theta \gamma^{a_{1} i_{1}} \theta\right)}{r^{9}} \\
& +\frac{F_{7} v_{i_{1}}(r \cdot v)\left(\epsilon \gamma^{a_{1}} \theta\right)\left(\theta \gamma^{a_{1} i_{1}} \theta\right)}{r^{9}}+\frac{F_{8} r_{i_{1}} r_{i_{2}} v_{i_{3}}(r \cdot v)\left(\epsilon \gamma^{a_{1} i_{1} i_{3}} \theta\right)\left(\theta \gamma^{a_{1} i_{2}} \theta\right)}{r^{11}} \\
& +\frac{F_{9} r_{i_{1}} r_{i_{2}} v_{i_{3}}(r \cdot v)\left(\epsilon \gamma^{i_{1}} \theta\right)\left(\theta \gamma^{i_{2} i_{3}} \theta\right)}{r^{11}}+\frac{F_{10} r_{i_{1}} v_{i_{2}} v_{i_{3}}\left(\epsilon \gamma^{a_{1} i_{1} i_{2}} \theta\right)\left(\theta \gamma^{a_{1} i_{3}} \theta\right)}{r^{9}} \\
& +\frac{F_{11} r_{i_{1}} v_{i_{2}} v_{i_{3}}\left(\epsilon \gamma^{i_{2}} \theta\right)\left(\theta \gamma^{i_{1} i_{3}} \theta\right)}{r^{9}}+\frac{F_{12} r_{i_{1}}(r \cdot v)\left(\epsilon \gamma^{a_{1} a_{2} i_{1}} \dot{\theta}\right)\left(\theta \gamma^{a_{1} a_{2}} \theta\right)}{r^{9}}+\frac{F_{13} v_{i_{1}}\left(\epsilon \gamma^{a_{1} a_{2} i_{1}} \dot{\theta}\right)\left(\theta \gamma^{a_{1} a_{2}} \theta\right)}{r^{7}} \\
& +\frac{F_{14} r_{i_{1}}(r \cdot v)\left(\epsilon \gamma^{a_{1}} \dot{\theta}\right)\left(\theta \gamma^{a_{1} i_{1}} \theta\right)}{r^{9}}+\frac{F_{15} r_{i_{1}} r_{i_{2}} v_{i_{3}}\left(\epsilon \gamma^{a_{1} i_{1} i_{3}} \dot{\theta}\right)\left(\theta \gamma^{a_{1} i_{2}} \theta\right)}{r^{9}}+\frac{F_{16} v_{i_{1}}\left(\epsilon \gamma^{a_{1}} \dot{\theta}\right)\left(\theta \gamma^{a_{1} i_{1}} \theta\right)}{r^{7}} \\
& +\frac{F_{17} r_{i_{1}} r_{i_{2}} v_{i_{3}}\left(\epsilon \gamma^{i_{1}} \dot{\theta}\right)\left(\theta \gamma^{i_{2} i_{3}} \theta\right)}{r^{9}}+\frac{F_{18} r_{i_{1}}(r \cdot v)\left(\epsilon \gamma^{a_{1} a_{2} a_{3} i_{1}} \dot{\theta}\right)\left(\theta \gamma^{a_{1} a_{2} a_{3}} \theta\right)}{r^{9}} \\
& +\frac{F_{19} v_{i_{1}}\left(\epsilon \gamma^{a_{1} a_{2} a_{3} i_{1}} \dot{\theta}\right)\left(\theta \gamma^{a_{1} a_{2} a_{3}} \theta\right)}{r^{7}}+\frac{F_{20} r_{i_{1}}(r \cdot v)\left(\epsilon \gamma^{a_{1} a_{2}} \dot{\theta}\right)\left(\theta \gamma^{a_{1} a_{2} i_{1}} \theta\right)}{r^{9}} \\
& +\frac{F_{21} r_{i_{1}} r_{i_{2}} v_{i_{3}}\left(\epsilon \gamma^{a_{1} a_{2} i_{1} i_{3}} \dot{\theta}\right)\left(\theta \gamma^{a_{1} a_{2} i_{2}} \theta\right)}{r^{9}}+\frac{F_{22} v_{i_{1}}\left(\epsilon \gamma^{a_{1} a_{2}} \dot{\theta}\right)\left(\theta \gamma^{a_{1} a_{2} i_{1}} \theta\right)}{r^{7}} \\
& +\frac{F_{23} r_{i_{1}} r_{i_{2}} v_{i_{3}}\left(\epsilon \gamma^{a_{1} i_{1}} \dot{\theta}\right)\left(\theta \gamma^{a_{1} i_{2} i_{3}} \theta\right)}{r^{9}}+\frac{F_{24} r_{i_{1}}\left(\epsilon \gamma^{a_{1} a_{2} i_{1}} \ddot{\theta}\right)\left(\theta \gamma^{a_{1} a_{2}} \theta\right)}{r^{7}}+\frac{F_{25} r_{i_{1}}\left(\epsilon \gamma^{a_{1}} \ddot{\theta}\right)\left(\theta \gamma^{a_{1} i_{1}} \theta\right)}{r^{7}} \\
& +\frac{F_{26} r_{i_{1}}\left(\epsilon \gamma^{a_{1} a_{2} a_{3} i_{1}} \ddot{\theta}\right)\left(\theta \gamma^{a_{1} a_{2} a_{3}} \theta\right)}{r^{7}}+\frac{F_{27} r_{i_{1}}\left(\epsilon \gamma^{a_{1} a_{2}} \ddot{\theta}\right)\left(\theta \gamma^{a_{1} a_{2} i_{1}} \theta\right)}{r^{7}}+\frac{F_{28} r_{i_{1}}\left(\epsilon \gamma^{a_{1} a_{2} i_{1}} \theta\right)\left(\dot{\theta} \gamma^{a_{1} a_{2}} \dot{\theta}\right)}{r^{7}} \\
& +\frac{F_{29} r_{i_{1}}\left(\epsilon \gamma^{a_{1}} \theta\right)\left(\dot{\theta} \gamma^{a_{1} i_{1}} \dot{\theta}\right)}{r^{7}}+\frac{F_{30} r_{i_{1}}\left(\epsilon \gamma^{a_{1} a_{2} a_{3} i_{1}} \theta\right)\left(\dot{\theta} \gamma^{a_{1} a_{2} a_{3}} \dot{\theta}\right)}{r^{7}}+\frac{F_{31} r_{i_{1}}\left(\epsilon \gamma^{a_{1} a_{2}} \theta\right)\left(\dot{\theta} \gamma^{a_{1} a_{2} i_{1}} \dot{\theta}\right)}{r^{7}} .
\end{aligned}
$$

Construction of $X_{\epsilon \theta^{6}}$ : The number of possible structures is 99 , which is rather non-trivial to generate. However, after proper use of Fierz identities, this gets drastically reduced to the following expression consisting of just 4 terms with arbitrary constants $G_{i}$ :

$$
\begin{aligned}
X_{\epsilon \theta^{7}}= & \frac{G_{1} r_{i_{1}}\left(\epsilon \gamma^{a_{1} a_{2}} \theta\right)\left(\theta \gamma^{a_{2} a_{3}} \theta\right)\left(\theta \gamma^{a_{3} a_{4}} \theta\right)\left(\theta \gamma^{a_{1} a_{4} i_{1}} \theta\right)}{r^{11}} \\
& +\frac{G_{2} r_{i_{1}} r_{i_{2}} r_{i_{3}}\left(\epsilon \gamma^{a_{1}} \theta\right)\left(\theta \gamma^{a_{1} i_{1}} \theta\right)\left(\theta \gamma^{a_{2} i_{2}} \theta\right)\left(\theta \gamma^{a_{2} i_{3}} \theta\right)}{r^{13}} \\
& +\frac{G_{3} r_{i_{1}} r_{i_{2}} r_{i_{3}}\left(\epsilon \gamma^{a_{1} i_{3}} \theta\right)\left(\theta \gamma^{a_{2} a_{3}} \theta\right)\left(\theta \gamma^{a_{3} i_{2}} \theta\right)\left(\theta \gamma^{a_{1} a_{2} i_{1}} \theta\right)}{r^{13}} \\
& +\frac{G_{4} r_{i_{1}} r_{i_{2}} r_{i_{3}}\left(\epsilon \gamma^{a_{1} a_{2}} \theta\right)\left(\theta \gamma^{a_{2} i_{3}} \theta\right)\left(\theta \gamma^{a_{3} i_{2}} \theta\right)\left(\theta \gamma^{a_{1} a_{3} i_{1}} \theta\right)}{r^{13}} .
\end{aligned}
$$

This completes the construction of the most general $X$ at order 3 . 


\subsection{Solution of the Ward identity}

We are now ready to solve the Ward identities (3.4) (3.8). Although in the end we will find that all the unknown coefficients vanish, we describe the consequence of each of these equations separately.

First consider (3.4). Since $X_{\epsilon \partial^{3} \theta}=0$ as we argued, we only need to take the appropriate SUSY variations of $\Delta \mathcal{L}_{\theta^{0}}^{(1)}$ and $\Delta \mathcal{L}_{\theta^{2}}^{(1)}$, given in (3.9) and (3.10), remove the total derivatives and set the result to zero. Collecting the structures of the same type, we get the equation

$$
\begin{aligned}
& 0=\delta_{r}^{(0)} \mathcal{L}_{\theta^{0}}^{(1)}+\delta_{\theta}^{(0)} \mathcal{L}_{\theta^{2}}^{(1)}=-\frac{\left(14 B_{5}+B_{6}-7 B_{9}-2 B_{10}\right) i r_{k} v_{i} a_{j}(r \cdot v)\left(\epsilon \gamma^{i j k} \theta\right)}{r^{9}} \\
& -\frac{\left(2 A_{6}+2 B_{1}\right) i \ddot{a}_{i}\left(\epsilon \gamma^{i} \theta\right)}{r^{5}}-\frac{\left(2 A_{4}-4 A_{5}-10 A_{6}-5 B_{1}-2 B_{4}-B_{9}-2 B_{11}\right) i a_{i} v^{2}\left(\epsilon \gamma^{i} \theta\right)}{r^{7}} \\
& -\frac{\left(-2 A_{3}-7 A_{4}-7 A_{5}+B_{6}-2 B_{8}\right) i r_{i} v^{4}\left(\epsilon \gamma^{i} \theta\right)}{r^{9}}-\frac{\left(2 A_{2}+2 A_{4}-5 A_{6}+2 B_{5}\right) i r_{i} a^{2}\left(\epsilon \gamma^{i} \theta\right)}{r^{7}} \\
& -\frac{\left(-2 A_{3}+70 A_{6}+35 B_{1}-2 B_{2}-B_{6}+7 B_{9}+2 B_{10}\right) i a_{i}(r \cdot v)^{2}\left(\epsilon \gamma^{i} \theta\right)}{r^{9}} \\
& -\frac{\left(-12 A_{1}+9 A_{3}+63 A_{4}-9 B_{6}-2 B_{7}\right) i r_{i} v^{2}(r \cdot v)^{2}\left(\epsilon \gamma^{i} \theta\right)}{r^{11}}-\frac{33 i A_{1} r_{i}(r \cdot v)^{4}\left(\epsilon \gamma^{i} \theta\right)}{r^{13}} \\
& -\frac{21 i A_{2} r_{i}(r \cdot a)^{2}\left(\epsilon \gamma^{i} \theta\right)}{r^{9}}-\frac{\left(-20 A_{6}-15 B_{1}-B_{9}\right) i \dot{a}_{i}(r \cdot v)\left(\epsilon \gamma^{i} \theta\right)}{r^{7}} \\
& -\frac{\left(-4 A_{3}-14 A_{4}+28 A_{5}-2 B_{2}+7 B_{4}-B_{6}+2 B_{8}\right) i v_{i} v^{2}(r \cdot v)\left(\epsilon \gamma^{i} \theta\right)}{r^{9}} \\
& -\frac{\left(18 A_{3}+9 B_{2}+9 B_{6}+2 B_{7}\right) i v_{i}(r \cdot v)^{3}\left(\epsilon \gamma^{i} \theta\right)}{r^{11}}-\frac{\left(4 A_{2}+2 A_{4}+2 B_{5}+B_{9}\right) i r_{i}(v \cdot \dot{a})\left(\epsilon \gamma^{i} \theta\right)}{r^{7}} \\
& -\frac{\left(4 A_{2}-2 A_{4}-10 A_{6}-5 B_{1}-2 B_{3}-2 B_{5}\right) i a_{i}(r \cdot a)\left(\epsilon \gamma^{i} \theta\right)}{r^{7}}-\frac{\left(-2 B_{5}+B_{9}\right) i r_{k} v_{i} \dot{a}_{j}\left(\epsilon \gamma^{i j k} \theta\right)}{r^{7}} \\
& -\frac{\left(-14 A_{2}-2 A_{3}-14 A_{4}+B_{6}\right) i r_{i} v^{2}(r \cdot a)\left(\epsilon \gamma^{i} \theta\right)}{r^{9}}-\frac{\left(-12 A_{1}+126 A_{2}\right) i r_{i}(r \cdot v)^{2}(r \cdot a)\left(\epsilon \gamma^{i} \theta\right)}{r^{11}} \\
& -\frac{\left(-28 A_{2}-4 A_{3}+14 A_{4}-2 B_{2}+7 B_{3}+14 B_{5}-B_{6}\right) i v_{i}(r \cdot v)(r \cdot a)\left(\epsilon \gamma^{i} \theta\right)}{r^{9}} \\
& -\frac{\left(4 A_{2}-2 A_{4}-B_{3}-2 B_{5}\right) i v_{i}(r \cdot \dot{a})\left(\epsilon \gamma^{i} \theta\right)}{r^{7}}+\frac{28 i A_{2} r_{i}(r \cdot v)(r \cdot \dot{a})\left(\epsilon \gamma^{i} \theta\right)}{r^{9}} \\
& -\frac{2 i A_{2} r_{i}(r \cdot \ddot{a})\left(\epsilon \gamma^{i} \theta\right)}{r^{7}}-\frac{\left(4 A_{2}+2 A_{4}-8 A_{5}-B_{3}-2 B_{4}+B_{9}+2 B_{11}\right) i v_{i}(v \cdot a)\left(\epsilon \gamma^{i} \theta\right)}{r^{7}} \\
& -\frac{\left(-28 A_{2}-4 A_{3}-28 A_{4}-14 B_{5}+B_{6}-7 B_{9}-2 B_{10}\right) i r_{i}(r \cdot v)(v \cdot a)\left(\epsilon \gamma^{i} \theta\right)}{r^{9}} .
\end{aligned}
$$

This produces 23 relations for 17 unknowns, which appears to be an overdetermined 
system. However, the solutions do exist and we obtain the following 15 relations:

$$
\begin{aligned}
& A_{1}=0, \quad A_{2}=0, \quad A_{3}=-\frac{7}{2} A_{4}, \quad A_{6}=\frac{A_{4}}{5}, \\
& B_{1}=\frac{A_{4}}{5}, \quad B_{2}=\frac{-7 A_{4}}{2}, \quad B_{3}=A_{4}, \quad B_{4}=-A_{4}+3 A_{5}, \quad B_{5}=\frac{A_{4}}{2}, \quad B_{6}=-7 A_{4}, \\
& B_{7}=\frac{63 A_{4}}{4}, \quad B_{8}=\frac{-7\left(A_{4}-A_{5}\right)}{2}, \quad B_{9}=A_{4}, \quad B_{10}=\frac{-7 A_{4}}{2}, \quad B_{11}=-A_{5} .
\end{aligned}
$$

Next we analyze the second Ward identity (3.5). Starting at this order, a large number of terms are produced after taking the relevant SUSY variations on the LHS, which are extremely difficult to fully simplify into independent structures by Fierz identities. Our strategy is to perform such simplification as much as possible and then use an explicit representation of the $S O(9) \gamma$-matrices to identify the coefficients of every independent structure written in terms of the components of $r^{m}, \theta^{\alpha}$ and their derivatives. In this way, the Ward identity is turned into a large set of coupled linear equations for the coefficients. Solving them with the aid of Mathematica, we found that all the coefficients involved in (3.5) vanish, namely,

$$
B_{i}=0,(i=1 \sim 11), \quad C_{j}=0,(j=1 \sim 13), \quad F_{k}=0,(k=1 \sim 31) .
$$

Together with the previous result (3.24), this implies that $A_{i}$ must all vanish as well.

The third Ward identity (3.6) was analyzed in an entirely similar fashion. The result is that again all the relevant coefficients must vanish:

$$
C_{i}=0,(i=1 \sim 13), \quad D_{j}=0,(j=1 \sim 4) .
$$

Likewise, the 4th Ward identity (3.7) turned out to dictate

$$
D_{i}=0,(i=1 \sim 4), \quad E_{j}=0,(j=1 \sim 3), \quad G_{k}=0,(k=1 \sim 4) .
$$

Finally, consider the last Ward identity (3.8). This is in fact the same as the relation studied in [9]. Our result agrees with [9] and gave the following relations among the coefficients $E_{i}$ :

$$
E_{1}=\frac{2}{143} E_{3}, \quad E_{2}=\frac{4}{13} E_{3}
$$

Since $E_{i}$ 's must vanish by the previous equation, this is just a consistency check.

Putting the results of all the analyses together, we found a remarkable fact

$$
\Delta \Gamma^{(1)}=0
$$


The precise meaning of this result is as follows: At 1-loop at order 4 in the derivative expansion, the solution to the Ward identity $\delta_{\epsilon} \Gamma=0$ regarded as a functional differential equation for $\Gamma$ is unique, provided that it exists. We would like to emphasize that, although we must assume the existence of the solution (which of course is guaranteed in Matrix theory), our demonstration does not make use of the explicit form of the quantum-corrected $\delta_{\epsilon}^{(1)}$ nor the knowledge of $\Gamma^{(1)}$ : Only the structure of the tree-level SUSY transformation law is relevant. We shall discuss the implication of this result further in the final section.

\section{Discussions}

Since the conclusion of our present study is extremely simple and clear, we shall in the following discuss its implications and make some remarks.

The fundamental question that we have been trying to answer in the series of investigations is how much of the structures of Matrix theory is governed by its symmetries, in particular by the supersymmetry. This requires the study of off-shell effective action $\Gamma$ as well, since at the quantum level the very notion of "on-shell" is defined by the full quantum-corrected equations of motion generated by $\Gamma$. What we have found in this work is that once we assume the existence of the quantum corrected SUSY transformation operator $\delta_{\epsilon}$, then the SUSY Ward identity, together with $S O(9)$ and CPT symmetries, dictates that, as far as the structures that would appear at 1-loop at order 4 in the system described by $r^{m}(\tau)$ and $\theta^{\alpha}(\tau)$ are concerned, fixes the off-shell effective action uniquely. The significance of our demonstration is that it does not refer to the underlying Matrix theory. This we believe is an important step in understanding the genuine power of maximal supersymmetry. As we have already mentioned in the introduction, our demonstration at order 4 is quite non-trivial since we know that from this order the real dynamics starts, namely that the S-matrix is non-trivial.

Would this property persist at higher orders? The fact that our logic was based solely on the tree-level SUSY transformation laws and that our result at order 4 is highly unlikely to be just an accident makes us feel that the answer ought to be yes. In fact we have already developed an analytic scheme by which such a proof appears feasible. The details will be presented in a forthcoming paper 15.

Finally, we wish to make a remark on the meaning of the real power of supersymmetry. Although we have demonstrated the uniqueness of the solution of the SUSY Ward identity, it is not enough to claim that SUSY determines the dynamics. Obviously we have not given prescriptions to compute $\delta_{\epsilon}$ and $\Gamma$ without referring to Matrix theory. If it is at 
all possible to prove such a claim, perhaps under some favorable conditions, one must study the closure property of the effective SUSY transformations, which inevitably gets intertwined with the knowledge of $\Gamma$ itself. In other words, $\delta_{\epsilon}$ and $\Gamma$ must be determined simultaneously in a self-consistent manner. Investigation of this problem is left for future study.

\section{Acknowledgment}

We thank H. Nicolai for clarifying comments. The research of Y.K. is supported in part by Grant-in-Aid for Scientific Research on Priority Area \#707 "Supersymmetry and Unified Theory of Elementary Particles" No. 10209204 and Grant-in-Aid for Scientific Research (B) No. 12440060, while that of T.M. is supported in part by the Japan Society for Promotion of Science under the Predoctoral Research Program No. 12-9617, all from the Japan Ministry of Education, Culture, Sports, Science and Technology. 


\section{Appendix: $\quad$ List of $S O(9)$ Fierz identities}

We list the $S O(9)$ Fierz identities used to simplify various expressions. They are presented in the order they are referred to in the text and classified by the number of uncontracted indices called "free indices".

\section{Identities used at $\mathcal{O}\left(\theta^{4}\right)$ :}

- 0-free-index type:

$$
\begin{aligned}
& \left(\theta \gamma^{a_{1} a_{2}} \theta\right)\left(\theta \gamma^{a_{1} a_{2}} \theta\right)=0, \quad\left(\theta \gamma^{a_{1} a_{2} a_{3}} \theta\right)\left(\theta \gamma^{a_{1} a_{2} a_{3}} \theta\right)=0 \\
& \left(\theta \gamma^{a_{1} a_{2}} \theta\right)\left(\dot{\theta} \gamma^{a_{1} a_{2}} \theta\right)=0, \quad\left(\theta \gamma^{a_{1} a_{2} a_{3}} \theta\right)\left(\dot{\theta} \gamma^{a_{1} a_{2} a_{3}} \theta\right)=0
\end{aligned}
$$

- 2-free-index type with an $\epsilon$-tensor:

$$
\left(\theta \gamma^{m_{1} m_{2} m_{3}} \theta\right)\left(\dot{\theta} \gamma^{a_{1} a_{2} a_{3} a_{4}} \theta\right) \epsilon_{a_{1} a_{2} a_{3} a_{4} i j m_{1} m_{2} m_{3}}=720(\dot{\theta} \theta)\left(\theta \gamma^{i j} \theta\right)
$$

- Another 0-free-index type

$$
\left(\dot{\theta} \gamma^{a_{1} a_{2}} \theta\right)\left(\dot{\theta} \gamma^{a_{1} a_{2}} \theta\right)=6(\dot{\theta} \theta)(\dot{\theta} \theta)+2\left(\dot{\theta} \gamma^{a_{1}} \theta\right)\left(\dot{\theta} \gamma^{a_{1}} \theta\right)
$$

- 2-free-index type

$$
\begin{aligned}
\left(\theta \gamma^{a_{1} a_{2} i} \theta\right)\left(\theta \gamma^{a_{1} a_{2} j} \theta\right) & =2\left(\theta \gamma^{a_{1} i} \theta\right)\left(\theta \gamma^{a_{1} j} \theta\right) \\
\left(\theta \gamma^{a_{1} a_{2} i} \theta\right)\left(\dot{\theta} \gamma^{a_{1} a_{2} j} \theta\right) & =8(\dot{\theta} \theta)\left(\theta \gamma^{i j} \theta\right)+2\left(\theta \gamma^{a_{1} j} \theta\right)\left(\dot{\theta} \gamma^{a_{1} i} \theta\right) \\
\left(\theta \gamma^{a_{1} i j} \theta\right)\left(\dot{\theta} \gamma^{a_{1}} \theta\right) & =\left(\theta \gamma^{a_{1} i} \theta\right)\left(\dot{\theta} \gamma^{a_{1} j} \theta\right)-3(\dot{\theta} \theta)\left(\theta \gamma^{i j} \theta\right)-\left(\theta \gamma^{a_{1} j} \theta\right)\left(\dot{\theta} \gamma^{a_{1} i} \theta\right) \\
\left(\theta \gamma^{a_{1} a_{2}} \theta\right)\left(\dot{\theta} \gamma^{a_{1} a_{2} i j} \theta\right)= & 2(\dot{\theta} \theta)\left(\theta \gamma^{i j} \theta\right)-2\left(\theta \gamma^{a_{1} j} \theta\right)\left(\dot{\theta} \gamma^{a_{1} i} \theta\right)+2\left(\theta \gamma^{a_{1} i} \theta\right)\left(\dot{\theta} \gamma^{a_{1} j} \theta\right) \\
\left(\theta \gamma^{a_{1} a_{2} j} \theta\right)\left(\dot{\theta} \gamma^{a_{1} a_{2} i} \dot{\theta}\right)= & -16\left(\dot{\theta} \gamma^{i} \theta\right)\left(\dot{\theta} \gamma^{j} \theta\right)+2\left(\theta \gamma^{a_{1} i} \theta\right)\left(\dot{\theta} \gamma^{a_{1} j} \dot{\theta}\right)-16(\dot{\theta} \theta)\left(\dot{\theta} \gamma^{i j} \theta\right) \\
& -4(\dot{\theta} \theta)(\dot{\theta} \theta) \delta_{i j}+4\left(\dot{\theta} \gamma^{a_{1}} \theta\right)\left(\dot{\theta} \gamma^{a_{1}} \theta\right) \delta_{i j} .
\end{aligned}
$$

- 4-free-index type:

$$
\begin{aligned}
\left(\theta \gamma^{a_{1} i j} \theta\right)\left(\theta \gamma^{a_{1} k l} \theta\right)= & 2\left(\theta \gamma^{i l} \theta\right)\left(\theta \gamma^{j k} \theta\right)-2\left(\theta \gamma^{i k} \theta\right)\left(\theta \gamma^{j l} \theta\right)-3\left(\theta \gamma^{i j} \theta\right)\left(\theta \gamma^{k l} \theta\right) \\
& +\left(\theta \gamma^{a_{1} j} \theta\right)\left(\theta \gamma^{a_{1} l} \theta\right) \delta_{i k}-\left(\theta \gamma^{a_{1} j} \theta\right)\left(\theta \gamma^{a_{1} k} \theta\right) \delta_{i l} \\
& -\left(\theta \gamma^{a_{1} i} \theta\right)\left(\theta \gamma^{a_{1} l} \theta\right) \delta_{j k}+\left(\theta \gamma^{a_{1} i} \theta\right)\left(\theta \gamma^{a_{1} k} \theta\right) \delta_{j l}
\end{aligned}
$$




\section{Identities used at $\mathcal{O}\left(\theta^{6}\right)$ :}

- 3-free-index type with an $\epsilon$-tensor:

$$
\begin{aligned}
&\left(\dot{\theta} \gamma^{a_{1} a_{2} a_{3}} \theta\right)(\left.\theta \gamma^{m_{1} m_{2} m_{3}} \theta\right) \epsilon_{a_{1} a_{2} a_{3} i j k m_{1} m_{2} m_{3}}=36\left(\dot{\theta} \gamma^{a_{1} i j} \theta\right)\left(\theta \gamma^{a_{1} k} \theta\right)+144\left(\dot{\theta} \gamma^{j} \theta\right)\left(\theta \gamma^{i k} \theta\right) \\
&-144\left(\dot{\theta} \gamma^{i} \theta\right)\left(\theta \gamma^{j k} \theta\right)-36\left(\dot{\theta} \gamma^{a_{1} k} \theta\right)\left(\theta \gamma^{a_{1} i j} \theta\right)+18\left(\dot{\theta} \gamma^{a_{1} a_{2}} \theta\right)\left(\theta \gamma^{a_{1} a_{2} j} \theta\right) \delta_{i k} \\
&-18\left(\dot{\theta} \gamma^{a_{1} a_{2}} \theta\right)\left(\theta \gamma^{a_{1} a_{2} i} \theta\right) \delta_{j k}, \\
&\left(\dot{\theta} \gamma^{a_{1} a_{2} a_{3} a_{4}} \theta\right)(\left.\theta \gamma^{m_{1} m_{2}} \theta\right) \epsilon_{a_{1} a_{2} a_{3} a_{4} i j k m_{1} m_{2}}=72\left(\dot{\theta} \gamma^{a_{1} i j} \theta\right)\left(\theta \gamma^{a_{1} k} \theta\right)+240\left(\dot{\theta} \gamma^{k} \theta\right)\left(\theta \gamma^{i j} \theta\right) \\
&+48\left(\dot{\theta} \gamma^{j} \theta\right)\left(\theta \gamma^{i k} \theta\right)-48\left(\dot{\theta} \gamma^{i} \theta\right)\left(\theta \gamma^{j k} \theta\right)-72\left(\dot{\theta} \gamma^{a_{1} k} \theta\right)\left(\theta \gamma^{a_{1} i j} \theta\right) \\
&+36\left(\dot{\theta} \gamma^{a_{1} a_{2}} \theta\right)\left(\theta \gamma^{a_{1} a_{2} j} \theta\right) \delta_{i k}-36\left(\dot{\theta} \gamma^{a_{1} a_{2}} \theta\right)\left(\theta \gamma^{a_{1} a_{2} i} \theta\right) \delta_{j k}, \\
&\left(\theta \gamma^{a_{1} a_{2} a_{3}} \theta\right)\left(\theta \gamma^{m_{1} m_{2} m_{3}} \theta\right) \epsilon_{a_{1} a_{2} a_{3} i j k m_{1} m_{2} m_{3}}=0 .
\end{aligned}
$$

- 4-free-index type with an $\epsilon$-tensor:

$$
\begin{aligned}
\left(\theta \gamma^{m_{1} m_{2}} \theta\right)\left(\theta \gamma^{a_{1} a_{2} a_{3}} \theta\right) \epsilon_{a_{1} a_{2} a_{3} i j k l m_{1} m_{2}}= & -24\left(\theta \gamma^{i l} \theta\right)\left(\theta \gamma^{j k} \theta\right)+24\left(\theta \gamma^{i k} \theta\right)\left(\theta \gamma^{j l} \theta\right) \\
& -24\left(\theta \gamma^{i j} \theta\right)\left(\theta \gamma^{k l} \theta\right) .
\end{aligned}
$$

- 5-free-index type with an $\epsilon$-tensor:

$$
\begin{aligned}
\left(\theta \gamma^{a_{1} a_{2} i} \theta\right) & \left(\theta \gamma^{m_{1} m_{2} m_{3}} \theta\right) \epsilon_{a_{1} a_{2} j k l m m_{1} m_{2} m_{3}}= \\
& -12\left(\theta \gamma^{l m} \theta\right)\left(\theta \gamma^{i j k} \theta\right)+12\left(\theta \gamma^{k m} \theta\right)\left(\theta \gamma^{i j l} \theta\right)-12\left(\theta \gamma^{k l} \theta\right)\left(\theta \gamma^{i j m} \theta\right) \\
& -12\left(\theta \gamma^{j m} \theta\right)\left(\theta \gamma^{i k l} \theta\right)+12\left(\theta \gamma^{j l} \theta\right)\left(\theta \gamma^{i k m} \theta\right)-12\left(\theta \gamma^{j k} \theta\right)\left(\theta \gamma^{i l m} \theta\right) \\
& -12\left(\theta \gamma^{i m} \theta\right)\left(\theta \gamma^{j k l} \theta\right)+12\left(\theta \gamma^{i l} \theta\right)\left(\theta \gamma^{j k m} \theta\right)-12\left(\theta \gamma^{i k} \theta\right)\left(\theta \gamma^{j l m} \theta\right) \\
& +12\left(\theta \gamma^{i j} \theta\right)\left(\theta \gamma^{k l m} \theta\right)
\end{aligned}
$$

- 1-free-index type:

$$
\left(\theta \gamma^{a_{1} a_{2} i} \theta\right)\left(\theta \gamma^{a_{1} a_{2}} \theta\right)=0 .
$$

- 3-free-index type:

$$
\begin{aligned}
\left(\theta \gamma^{a_{1} a_{2} k} \theta\right)\left(\dot{\theta} \gamma^{a_{1} a_{2} i j} \theta\right) & =-8\left(\theta \gamma^{j k} \theta\right)\left(\dot{\theta} \gamma^{i} \theta\right)+8\left(\theta \gamma^{i k} \theta\right)\left(\dot{\theta} \gamma^{j} \theta\right)+10\left(\theta \gamma^{i j} \theta\right)\left(\dot{\theta} \gamma^{k} \theta\right) \\
& -2\left(\theta \gamma^{a_{1} i j} \theta\right)\left(\dot{\theta} \gamma^{a_{1} k} \theta\right)+2\left(\theta \gamma^{a_{1} k} \theta\right)\left(\dot{\theta} \gamma^{a_{1} i j} \theta\right) \\
& +2\left(\theta \gamma^{a_{1} a_{2} j} \theta\right)\left(\dot{\theta} \gamma^{a_{1} a_{2}} \theta\right) \delta_{i k}-2\left(\theta \gamma^{a_{1} a_{2} i} \theta\right)\left(\dot{\theta} \gamma^{a_{1} a_{2}} \theta\right) \delta_{j k} .
\end{aligned}
$$


- 4-free-index type:

$$
\begin{aligned}
\left(\theta \gamma^{a_{1} i j} \theta\right)\left(\dot{\theta} \gamma^{a_{1} k l} \theta\right)= & -\left(\theta \gamma^{j k l} \theta\right)\left(\dot{\theta} \gamma^{i} \theta\right)+\left(\theta \gamma^{i k l} \theta\right)\left(\dot{\theta} \gamma^{j} \theta\right)+\left(\theta \gamma^{i j l} \theta\right)\left(\dot{\theta} \gamma^{k} \theta\right) \\
& -\left(\theta \gamma^{i j k} \theta\right)\left(\dot{\theta} \gamma^{l} \theta\right)-2\left(\theta \gamma^{k l} \theta\right)\left(\dot{\theta} \gamma^{i j} \theta\right)-\left(\theta \gamma^{j l} \theta\right)\left(\dot{\theta} \gamma^{i k} \theta\right) \\
& +\left(\theta \gamma^{j k} \theta\right)\left(\dot{\theta} \gamma^{i l} \theta\right)+\left(\theta \gamma^{i l} \theta\right)\left(\dot{\theta} \gamma^{j k} \theta\right)-\left(\theta \gamma^{i k} \theta\right)\left(\dot{\theta} \gamma^{j l} \theta\right) \\
& -\left(\theta \gamma^{i j} \theta\right)\left(\dot{\theta} \gamma^{k l} \theta\right)-(\dot{\theta} \theta)\left(\theta \gamma^{j l} \theta\right) \delta_{i k}-\left(\theta \gamma^{a_{1} j l} \theta\right)\left(\dot{\theta} \gamma^{a_{1}} \theta\right) \delta_{i k} \\
& +\left(\theta \gamma^{a_{1} j} \theta\right)\left(\dot{\theta} \gamma^{a_{1} l} \theta\right) \delta_{i k}+(\dot{\theta} \theta)\left(\theta \gamma^{j k} \theta\right) \delta_{i l}+\left(\theta \gamma^{a_{1} j k} \theta\right)\left(\dot{\theta} \gamma^{a_{1}} \theta\right) \delta_{i l} \\
& -\left(\theta \gamma^{a_{1} j} \theta\right)\left(\dot{\theta} \gamma^{a_{1} k} \theta\right) \delta_{i l}+(\dot{\theta} \theta)\left(\theta \gamma^{i l} \theta\right) \delta_{j k}+\left(\theta \gamma^{a_{1} i l} \theta\right)\left(\dot{\theta} \gamma^{a_{1}} \theta\right) \delta_{j k} \\
& -\left(\theta \gamma^{a_{1} i} \theta\right)\left(\dot{\theta} \gamma^{a_{1} l} \theta\right) \delta_{j k}-(\dot{\theta} \theta)\left(\theta \gamma^{i k} \theta\right) \delta_{j l}-\left(\theta \gamma^{a_{1} i k} \theta\right)\left(\dot{\theta} \gamma^{a_{1}} \theta\right) \delta_{j l} \\
& +\left(\theta \gamma^{a_{1} i} \theta\right)\left(\dot{\theta} \gamma^{a_{1} k} \theta\right) \delta_{j l} .
\end{aligned}
$$

\section{Identities used for total derivative terms:}

- 1-free-index type:

$$
\begin{aligned}
\left(\epsilon \gamma^{a_{1} a_{2}} \theta\right)\left(\theta \gamma^{a_{1} a_{2} i} \theta\right) & =-2\left(\epsilon \gamma^{a_{1}} \theta\right)\left(\theta \gamma^{a_{1} i} \theta\right), \\
\left(\epsilon \gamma^{a_{1} a_{2} i} \theta\right)\left(\theta \gamma^{a_{1} a_{2}} \theta\right) & =+2\left(\epsilon \gamma^{a_{1}} \theta\right)\left(\theta \gamma^{a_{1} i} \theta\right), \\
\left(\epsilon \gamma^{a_{1} a_{2} a_{3} i} \theta\right)\left(\theta \gamma^{a_{1} a_{2} a_{3}} \theta\right) & =-6\left(\epsilon \gamma^{a_{1}} \theta\right)\left(\theta \gamma^{a_{1} i} \theta\right) .
\end{aligned}
$$

- 3-free-index type:

$$
\begin{aligned}
\left(\epsilon \gamma^{a_{1} a_{2} i j} \theta\right)\left(\theta \gamma^{a_{1} a_{2} k} \theta\right)= & 2\left(\epsilon \gamma^{a_{1} i j} \theta\right)\left(\theta \gamma^{a_{1} k} \theta\right)+10\left(\epsilon \gamma^{k} \theta\right)\left(\theta \gamma^{i j} \theta\right)+8\left(\epsilon \gamma^{j} \theta\right)\left(\theta \gamma^{i k} \theta\right) \\
& -8\left(\epsilon \gamma^{i} \theta\right)\left(\theta \gamma^{j k} \theta\right)-2\left(\epsilon \gamma^{a_{1} k} \theta\right)\left(\theta \gamma^{a_{1} i j} \theta\right)-4\left(\epsilon \gamma^{a_{1}} \theta\right)\left(\theta \gamma^{a_{1} j} \theta\right) \delta_{i k} \\
& +4\left(\epsilon \gamma^{a_{1}} \theta\right)\left(\theta \gamma^{a_{1} i} \theta\right) \delta_{j k}, \\
\left(\epsilon \gamma^{a_{1} k} \theta\right)\left(\theta \gamma^{a_{1} i j} \theta\right)= & -\left(\epsilon \gamma^{a_{1} j k} \theta\right)\left(\theta \gamma^{a_{1} i} \theta\right)+\left(\epsilon \gamma^{a_{1} i k} \theta\right)\left(\theta \gamma^{a_{1} j} \theta\right)+3\left(\epsilon \gamma^{k} \theta\right)\left(\theta \gamma^{i j} \theta\right) \\
& +\left(\epsilon \gamma^{j} \theta\right)\left(\theta \gamma^{i k} \theta\right)-\left(\epsilon \gamma^{i} \theta\right)\left(\theta \gamma^{j k} \theta\right)+(\epsilon \theta)\left(\theta \gamma^{i j k} \theta\right) \\
& -\left(\epsilon \gamma^{a_{1}} \theta\right)\left(\theta \gamma^{a_{1} j} \theta\right) \delta_{i k}+\left(\epsilon \gamma^{a_{1}} \theta\right)\left(\theta \gamma^{a_{1} i} \theta\right) \delta_{j k} .
\end{aligned}
$$




\section{References}

[1] Y. Kazama and T. Muramatsu, Nucl. Phys. 584 (2000) 171, hep-th/0003161.

[2] Y. Kazama and T. Muramatsu, Class. Quant. Grav. 18, 2277 (2001), hep-th/0103116.

[3] T. Banks, W. Fischler, S. H. Shenker and L. Susskind, Phys. Rev. D55 (1997) 5112, hep-th/9610043.

[4] L. Susskind, hep-th/9704080.

[5] For pioneering works, see M. Claudson and M. B. Halpern, Nucl. Phys. B 250, 689 (1985); R. Flume, Annals Phys. 164, 189 (1985); M. Baake, M. Reinicke and V. Rittenberg, J. Math. Phys. 26, 1070 (1985); B. de Wit, J. Hoppe and H. Nicolai, Nucl. Phys. B 305, 545 (1988).

[6] T. Banks, Nucl. Phys. Proc. Suppl. 67, 180 (1998), hep-th/9710231; T. Banks, hep-th/9911068; D. Bigatti and L. Susskind, hep-th/9712072; A. Bilal, Fortsch. Phys. 47, 5 (1999), hep-th/9710136; B. de Wit, hep-th/9902051; A. Konechny and A. Schwarz, hep-th/0012145; H. Nicolai and R. Helling, hep-th/9809103; N. A. Obers and B. Pioline, Phys. Rept. 318, 113 (1999), hep-th/9809039; W. Taylor, hep-th/9801182; W. Taylor, hep-th/0002016; W. Taylor, hep-th/0101126.

[7] N. Seiberg, Phys. Rev. Lett. 79 (1997) 3577, hep-th/9710009.

[8] A. Sen, Adv. Theor. Math. Phys. 2 (1998) 51, hep-th/9709220.

[9] S. Paban, S. Sethi and M. Stern, Nucl. Phys. B534 (1998) 137, hep-th/9805018.

[10] S. Paban, S. Sethi and M. Stern, J. High Energy Phys. 06 (1998) 012, hepth/9806028.

[11] D. A. Lowe, J. High Energy Phys. 11 (1998) 009, hep-th/9810075.

[12] S. Sethi and M. Stern, J. High Energy Phys. 06 (1999) 004, hep-th/9903049.

[13] S. Hyun, Y. Kiem and H. Shin, Nucl. Phys. B558 (1999) 349, hep-th/9903022.

[14] H. Nicolai and J. Plefka, Phys. Lett. B477 (2000) 309, hep-th/0001106.

[15] Y. Kazama and T. Muramatsu, work in progress. 